\title{
Mean-strain 8-node Hexahedron with Optimized Energy-Sampling Stabilization
}

\author{
P. Krysl ${ }^{\mathrm{a}, 1}$, \\ ${ }^{a}$ University of California, San Diego, 9500 Gilman Dr., \#0085, La Jolla, CA 92093
}

\begin{abstract}
A method for stabilizing the mean-strain hexahedron was described by Krysl (in IJNME 2014). The technique relied on a sampling of the stabilization energy using the mean-strain quadrature and the full Gaussian integration rule, which was shown to guarantee consistency and stability. The stabilization energy was assumed to be generated by a modified constitutive matrix based on the spectral decomposition. The stabilization required user-selected values of the stabilization parameters. In the present work we eliminate the arbitrariness of the stabilization parameters. We formulate the technique more precisely as an assumed-strain method, and we express the stabilization energy in terms of input parameters of the real material. Finally, we fix the value of the stabilization parameters in a quasi-optimal manner by linking the stabilization to the bending behavior of the hexahedral element. For simplicity the developments are limited to linear elasticity, but with an arbitrarily anisotropic elasticity matrix. The accuracy and convergence characteristics of the present formulations compare favorably with the capabilities of mean-strain and other high-performance hexahedral elements as implemented in Abaqus and with a number of successful hexahedral and shell elements and we demonstrate that the present element performs very well when used with large aspect ratios for thin structures such as plates or shells.
\end{abstract}

Keywords: anisotropic elasticity, nearly incompressible, mean-strain hexahedron, uniform strain hexahedron, finite element

PACS: ???

\section{Introduction}

First-order bricks (hexahedra) tend to be exploited for 3-D analysis for their efficiency, robustness, and ease-of-use. Eight-node mean-strain hexahedra seem to be capable of providing both coarse-mesh accuracy and reliable convergence, and significant progress has been made over the years in this technology.

We refer to $[1,2]$ for overview of the recent developments. The main issues for the mean-strain hexahedra are how to achieve at the same time (i) locking-free

Email address: pkrysl@ucsd.edu (P. Krysl)

Preprint submitted to Elsevier

August 12, 2015 
response, (ii) good coarse-mesh accuracy, and (iii) stability. Strictly mean-strain hexahedra achieve locking-free response, but lose stability. Adding stability, for instance by treating the so-called hourglassing modes, would tend to affect locking and accuracy. Coarse-mesh accuracy requires of the stabilization to not to deteriorate the response of the element but rather to enhance the ability of the element to accurately respond to deformations in the hourglassing modes such as bending or torsion. Puso's hexahedron is a good example of a successful approach [3].

A method for stabilizing the mean-strain hexahedron that differed from the then-known approaches was described by Krysl [4]. The technique relied on a sampling of the stabilization energy using two quadrature rules, the mean-strain quadrature and the full Gaussian integration rule. The use of two quadrature rules was shown to guarantee both consistency and elimination of the hourglassing modes. The stabilization energy was assumed to be generated by a modified constitutive matrix based on the spectral decomposition. The stabilization required user-selected values of stabilization parameters, which is in general undesirable.

In the present work we eliminate the arbitrariness of the stabilization parameters. Firstly, in Section 1 we formulate the technique more precisely as an assumed-strain method. The stabilization energy is then introduced in Section 2 as a quadratic form which is added and subtracted at the same time: added for strains linked to the displacements and subtracted for the assumed strains. We develop an argument for the resulting hexahedral element being convergent by establishing consistency and positive-semi-definiteness of the strain energy. In Section 3 the parameters of the stabilization material are expressed in terms of input parameters of the real material in a way that avoids locking due to volumetric and other constraints (such as for strongly anisotropic materials). The value of the remaining stabilization parameter (Young's modulus) is fixed in a quasi-optimal manner by linking the stabilization to the bending behavior of the hexahedral element. For simplicity the developments are limited to linear elasticity, but with an arbitrarily anisotropic elasticity matrix.

Section 4 illustrates the performance of the proposed approaches on a variety of benchmark problems, for isotropic and anisotropic material models. Importantly, the coarse-mesh response is significantly improved by the choice of the stabilization parameter. This proves important especially for thin shells and plates, where the present element is shown to match the performance of specialized shell and plate elements. The performance of the stabilization is also tested for highly distorted elements in a vibration problem. The present stabilization technique is also shown to work for anisotropic materials.

The accuracy and convergence characteristics of the present formulations compare favorably with the capabilities of mean-strain and other high-performance hexahedral elements as implemented in Abaqus. In addition, we compare with a number of successful hexahedral and shell elements and we demonstrate that the present element performs very well for thin structures such as plates or shells. Crucially, the hexahedron formulation presented here eliminates the need for user-selected values of the stabilization parameters. Together with excellent 
performance this makes the present element a good general-purpose hexahedron.

\section{Assumed-strain formulation}

We will re-derive the mean-strain eight-node hexahedra $[5,6]$ taking the variational approach. This will make the inclusion of the stabilization particularly illuminating. We shall take as the starting point the strain-displacement (de Veubeke) functional

$$
\Pi(\overline{\boldsymbol{\epsilon}}, \boldsymbol{u})=\Psi(\overline{\boldsymbol{\epsilon}})+\int_{\Omega} \overline{\boldsymbol{\sigma}} \cdot(\boldsymbol{\epsilon}-\overline{\boldsymbol{\epsilon}}) d \Omega-\mathcal{W} .
$$

Here

$$
\Psi(\overline{\boldsymbol{\epsilon}})=\int_{\Omega} \mathcal{U}(\overline{\boldsymbol{\epsilon}}) d \Omega,
$$

is the strain energy, where the energy density $\mathcal{U}(\overline{\boldsymbol{\epsilon}})$ is generated by the assumed strains $\overline{\boldsymbol{\epsilon}}$. For instance in linear elasticity we define a quadratic form of the assumed strains $\overline{\boldsymbol{\epsilon}}$

$$
\mathcal{U}(\overline{\boldsymbol{\epsilon}})=\frac{1}{2} \overline{\boldsymbol{\epsilon}}^{T} \mathbf{D} \overline{\boldsymbol{\epsilon}}
$$

with a symmetric positive definite constitutive matrix $\mathbf{D}$. The strains are represented here $\boldsymbol{\epsilon}=\left[\epsilon_{11}, \epsilon_{22}, \epsilon_{33}, 2 \epsilon_{32}, 2 \epsilon_{31}, 2 \epsilon_{12}\right]^{T}$ in the Voigt-Mandel representation of the second order strain tensor (and analogously for $\bar{\epsilon}$ ).

We shall assume that the material properties $\mathbf{D}$ and the assumed strains $\overline{\boldsymbol{\epsilon}}$ within each hexahedral element are uniform. The stress $\bar{\sigma}$ is obtained as

$$
\bar{\sigma}=\frac{\partial \mathcal{U}(\overline{\boldsymbol{\epsilon}})}{\partial \bar{\epsilon}} .
$$

Now we shall express the first variation of the functional (1) as

$$
\delta \Pi(\overline{\boldsymbol{\epsilon}}, \boldsymbol{u})=\int_{\Omega}\left[\frac{\partial \mathcal{U}(\overline{\boldsymbol{\epsilon}})}{\partial \overline{\boldsymbol{\epsilon}}} \cdot \delta \overline{\boldsymbol{\epsilon}}+\delta \overline{\boldsymbol{\sigma}} \cdot(\boldsymbol{\epsilon}-\overline{\boldsymbol{\epsilon}})+\overline{\boldsymbol{\sigma}} \cdot(\delta \boldsymbol{\epsilon}-\delta \overline{\boldsymbol{\epsilon}})\right] d \Omega-\delta \mathcal{W},
$$

where the variation of the stress may be expressed directly as

$$
\delta \overline{\boldsymbol{\sigma}}=\frac{\partial^{2} \mathcal{U}(\overline{\boldsymbol{\epsilon}})}{\partial \overline{\boldsymbol{\epsilon}}^{2}} \cdot \delta \overline{\boldsymbol{\epsilon}}
$$

The matrix of tangent moduli may be identified as

$$
\mathbf{D}=\frac{\partial^{2} \mathcal{U}(\overline{\boldsymbol{\epsilon}})}{\partial \overline{\boldsymbol{\epsilon}}^{2}} .
$$

As usual, the solution to (5) will follow from

$$
\delta \Pi(\overline{\boldsymbol{\epsilon}}, \boldsymbol{u})=0,
$$


but we will achieve this by separating out the second and third term that link the displacement-related strains and the assumed strains so that we will require the satisfaction of the balance equation

$$
\int_{\Omega} \frac{\partial \mathcal{U}(\overline{\boldsymbol{\epsilon}})}{\partial \overline{\boldsymbol{\epsilon}}} \cdot \delta \overline{\boldsymbol{\epsilon}} d \Omega-\delta \mathcal{W}=0
$$

and, separately, the satisfaction of the kinematic equation

$$
\int_{\Omega} \delta \overline{\boldsymbol{\epsilon}} \cdot \mathbf{D} \cdot(\boldsymbol{\epsilon}-\overline{\boldsymbol{\epsilon}})+\overline{\boldsymbol{\sigma}} \cdot(\delta \boldsymbol{\epsilon}-\delta \overline{\boldsymbol{\epsilon}}) d \Omega=0 .
$$

The form of the assumed strains will be derived from (10).

At this point we introduce the finite element approximation. Expression (10) is evaluated by adding contributions from individual finite elements. Therefore (10) may be specialized to the domain of a single hexahedral finite element $\Omega^{(e)}$ as

$$
\int_{\Omega^{(e)}} \delta \overline{\boldsymbol{\epsilon}} \cdot \mathbf{D} \cdot(\boldsymbol{\epsilon}-\overline{\boldsymbol{\epsilon}})+\overline{\boldsymbol{\sigma}} \cdot(\delta \boldsymbol{\epsilon}-\delta \overline{\boldsymbol{\epsilon}}) d \Omega=0 .
$$

Here we express the strain vectors in dependence on the displacement in the form

$$
\boldsymbol{\epsilon}=\mathbf{B} \boldsymbol{U} \quad \text { and } \quad \bar{\epsilon}=\overline{\mathbf{B}} \boldsymbol{U}
$$

where the strain-displacement matrix $\mathbf{B}$ is the usual expression calculated from the gradients of the basis functions, the $24 \times 1$ vector $\boldsymbol{U}$ consists of the nodal displacements,

$$
\boldsymbol{U}=\left[\boldsymbol{u}_{1}, \boldsymbol{u}_{2}, \ldots, \boldsymbol{u}_{8}\right]^{T}
$$

and the assumed strain-displacement matrix $\overline{\mathbf{B}}$ will be derived below.

Substitution of (12) into (11) leads to

$$
\int_{\Omega^{(e)}}(\mathbf{D} \overline{\mathbf{B}} \delta \boldsymbol{U}) \cdot(\mathbf{B}-\overline{\mathbf{B}}) \boldsymbol{U}+\overline{\boldsymbol{\sigma}} \cdot(\mathbf{B}-\overline{\mathbf{B}}) \delta \boldsymbol{U} d \Omega=0 .
$$

Extricating the nodal displacement vectors from underneath the integral signs we obtain

$$
\delta \boldsymbol{U} \cdot \int_{\Omega^{(e)}}(\mathbf{D} \overline{\mathbf{B}}) \cdot(\mathbf{B}-\overline{\mathbf{B}}) d \Omega \cdot \boldsymbol{U}+\int_{\Omega^{(e)}} \overline{\boldsymbol{\sigma}} \cdot(\mathbf{B}-\overline{\mathbf{B}}) d \Omega \cdot \delta \boldsymbol{U}=0 .
$$

If we recollect now that $\overline{\boldsymbol{\sigma}}, \mathbf{D}$, and $\overline{\mathbf{B}}$ are uniform over the domain of the element, we are immediately led for each element $e$ to the condition

$$
\delta \boldsymbol{U} \cdot \mathbf{D} \cdot \overline{\mathbf{B}} \cdot \int_{\Omega^{(e)}}(\mathbf{B}-\overline{\mathbf{B}}) d \Omega \cdot \boldsymbol{U}+\overline{\boldsymbol{\sigma}} \cdot \int_{\Omega^{(e)}}(\mathbf{B}-\overline{\mathbf{B}}) d \Omega \cdot \delta \boldsymbol{U}=0,
$$

which may be satisfied by taking

$$
\int_{\Omega^{(e)}}(\mathbf{B}-\overline{\mathbf{B}}) d \Omega=\mathbf{0} .
$$


Thus we are then in a position to define the assumed strain-displacement matrix as

$$
\overline{\mathbf{B}}=\left(\int_{\Omega^{(e)}} d \Omega\right)^{-1} \int_{\Omega^{(e)}} \mathbf{B} d \Omega=V_{\mathrm{e}}^{-1} \int_{\Omega^{(e)}} \mathbf{B} d \Omega .
$$

Here $V_{\mathrm{e}}$ is the element volume. The assumed strain-displacement matrix is the mean of the strain-displacement matrix $\mathbf{B}$ over the domain of the element $e$, hence the designation of the formulation as the mean-strain approach.

The strain energy function (2) modified by the steps above thus reads for a single element

$$
\bar{\Psi}_{e}(\boldsymbol{U})=\int_{\Omega} \mathcal{U}(\overline{\boldsymbol{\epsilon}}) d \Omega=V_{e} \mathcal{U}(\overline{\boldsymbol{\epsilon}}) \quad \text { where } \overline{\boldsymbol{\epsilon}}=\overline{\mathbf{B}} \boldsymbol{U} .
$$

Due to the vanishing of the second term, the functional of equation (1) can be simplified with the introduction of the modified strain energy as

$$
\bar{\Pi}(\boldsymbol{u})=\bar{\Psi}(\boldsymbol{u})-\mathcal{W} .
$$

We have thus obtained a single-field functional.

The stiffness matrix of a single element resulting from the first variation of (20) reads

$$
\mathbf{K}_{\mathrm{e}, \mathrm{ms}}=V_{\mathrm{e}} \overline{\mathbf{B}}^{T} \mathbf{D} \overline{\mathbf{B}}
$$

and has a rank of six, generated by the constant-strain modes. The defect of this matrix is therefore equal to 12 , and the formulation needs to be stabilized $[7,5$, $6]$.

\section{Stabilization}

Our approach to the stabilization differs from the well-known approaches of Flanagan and Belytschko [5, 2] (perturbation hourglassing stabilization); of Belytschko and Bindemann [6] (assumed-strain stabilization), and Puso [3] and Reese [8]; of Reese [9] (enhanced-strain (incompatible mode) stabilization); and of Belytschko and Bachrach [10] and Liu et al. [11] (physical stabilization based on the Taylor-series expansion of the strains). We propose the so-called stabilization by energy sampling [4].

The energy density to be sampled is generated by a positive-definite constitutive matrix $\widehat{\mathbf{D}}$ in the quadratic form

$$
\widehat{\mathcal{U}}(\boldsymbol{y})=\frac{1}{2} \boldsymbol{y}^{T} \widehat{\mathbf{D}} \boldsymbol{y} .
$$

We will refer to $\widehat{\mathcal{U}}$ as the stabilization energy density. The strain energy of each element $e(19)$ will be modified by the simultaneous addition and subtraction of the stabilization energy to read

$$
\bar{\Psi}_{e}(\boldsymbol{U})=\int_{\Omega} \mathcal{U}(\overline{\boldsymbol{\epsilon}}) d \Omega+\int_{\Omega} \widehat{\mathcal{U}}(\boldsymbol{\epsilon}) d \Omega-\int_{\Omega} \widehat{\mathcal{U}}(\overline{\boldsymbol{\epsilon}}) d \Omega,
$$


where, importantly, the stabilization energy is generated by either the displacementrelated strains $\boldsymbol{\epsilon}$ or by the assumed strains $\overline{\boldsymbol{\epsilon}}$ (both depend on the displacement $\boldsymbol{U}$ as outlined above).

The sampling of the energy by numerical quadrature is now carefully chosen to ensure both convergence to the correct solution and stability. The integral over the hexahedral element of the stabilization energy associated with displacement-derived strains $\epsilon$

$$
\int_{\Omega} \widehat{\mathcal{U}}(\boldsymbol{\epsilon}) d \Omega
$$

will be evaluated with the full $2 \times 2 \times 2$ Gauss quadrature. The integral of $\widehat{\mathcal{U}}(\overline{\boldsymbol{\epsilon}})$ over the hexahedral element will be evaluated with the mean-strain quadrature as

$$
\int_{\Omega} \widehat{\mathcal{U}}(\overline{\boldsymbol{\epsilon}}) d \Omega=V_{\mathrm{e}} \widehat{\mathcal{U}}(\overline{\boldsymbol{\epsilon}})
$$

As a result of this construction we can prevent hourglass modes and we maintain consistency.

\subsection{Consistency}

The stiffness matrix (21) derives its properties from the strain-displacement matrix (18). This matrix is capable of generating uniform strains from displacements of the nodes $k$ that are elements of the set of linear deformations

$$
\{\boldsymbol{U}\}_{L I N}=\left\{\boldsymbol{U}=\left[\boldsymbol{u}_{k}\right]: \boldsymbol{u}_{k}=\boldsymbol{u}\left(\boldsymbol{x}_{k}\right)=\mathbf{E}_{L I N} \boldsymbol{x}_{k}\right\},
$$

where the symmetric matrix $\mathbf{E}_{L I N}$ consists of the Voigt components of the uniform strains

$$
\mathbf{E}_{L I N}=\left[\begin{array}{ccc}
\bar{\epsilon}_{x} & \bar{\epsilon}_{x y} / 2 & \bar{\epsilon}_{x z} / 2 \\
\bar{\epsilon}_{x y} / 2 & \bar{\epsilon}_{y} & \bar{\epsilon}_{y z} / 2 \\
\bar{\epsilon}_{x z} / 2 & \bar{\epsilon}_{y z} / 2 & \bar{\epsilon}_{z}
\end{array}\right] .
$$

The strain displacement matrix $\mathbf{B}(\boldsymbol{x})$ in the expression for the stabilization strain energy from the displacement-derived strains

$$
\int_{\Omega} \widehat{\mathcal{U}}(\boldsymbol{\epsilon}) d \Omega=\frac{1}{2} \int_{\Omega} \boldsymbol{u}^{T} \mathbf{B}^{T} \widehat{\mathbf{D}} \mathbf{B} \boldsymbol{u} d \Omega
$$

will also produce uniform strains from the displacements (26). Consequently the two stabilization energy contributions will cancel for the linear displacement (26)

$$
-V_{\mathrm{e}} \widehat{\mathcal{U}}(\overline{\boldsymbol{\epsilon}})+\int_{\Omega} \widehat{\mathcal{U}}(\boldsymbol{\epsilon}) d \Omega=0 \quad \text { for } \boldsymbol{U} \in\{\boldsymbol{U}\}_{L I N} .
$$

The entire contribution to the strain energy is then due to the real material

$$
\bar{\Psi}_{e}(\boldsymbol{U})=V_{\mathrm{e}} \mathcal{U}(\overline{\boldsymbol{\epsilon}}) \quad \text { for } \boldsymbol{U} \in\{\boldsymbol{U}\}_{L I N} .
$$


In addition to the nodal displacement vector corresponding to the uniform strains (26) consider also the nodal displacements produced by rigid body displacements

$$
\{\boldsymbol{U}\}_{R B M}=\left\{\boldsymbol{U}=\left[\boldsymbol{u}_{k}\right]: \boldsymbol{u}_{k}=\boldsymbol{u}\left(\boldsymbol{x}_{k}\right)=\boldsymbol{a}+\boldsymbol{\Omega}_{R B M} \boldsymbol{x}_{k}\right\},
$$

where $\boldsymbol{a}$ is the rigid body translation and the skew-symmetric matrix $\boldsymbol{\Omega}_{R B M}$ produces rigid body rotations. Note that the strain energy contributions all vanish for rigid body displacements

$$
\bar{\Psi}_{e}(\boldsymbol{U})=V_{\mathrm{e}} \mathcal{U}(\overline{\boldsymbol{\epsilon}})=V_{\mathrm{e}} \widehat{\mathcal{U}}(\overline{\boldsymbol{\epsilon}})=\int_{\Omega} \widehat{\mathcal{U}}(\boldsymbol{\epsilon}) d \Omega=0 \quad \text { for } \boldsymbol{U} \in\{\boldsymbol{U}\}_{R B M} .
$$

The above findings show the consistency of the method.

\subsection{Suppression of hourglassing}

The set of all displacements in the set $\{\boldsymbol{U}\}_{H G}$ such that

$$
\{\boldsymbol{U}\}_{H G} \cap\{\boldsymbol{U}\}_{L I N}=\emptyset
$$

and

$$
\{\boldsymbol{U}\}_{H G} \cap\{\boldsymbol{U}\}_{R B M}=\emptyset
$$

are the hourglassing deformations. The strain energy contributions obtained from the corresponding assumed strains both vanish

$$
V_{\mathrm{e}} \mathcal{U}(\overline{\boldsymbol{\epsilon}})=V_{\mathrm{e}} \widehat{\mathcal{U}}(\overline{\boldsymbol{\epsilon}})=0 \quad \text { for } \boldsymbol{U} \in\{\boldsymbol{U}\}_{H G},
$$

but the $2 \times 2 \times 2$ Gaussian quadrature yields

$$
\bar{\Psi}_{e}(\boldsymbol{U})=\int_{\Omega} \widehat{\mathcal{U}}(\boldsymbol{\epsilon}) d \Omega>0 \quad \text { for } \boldsymbol{U} \in\{\boldsymbol{U}\}_{H G} .
$$

Consequently, the strain energy $\bar{\Psi}_{e}(\boldsymbol{U})$ is positive semi-definite, and it becomes zero only for rigid body displacements.

The consistency and the properties of the modified strain energy (23) thus show that the element passes all three versions of the patch test [12] and is therefore convergent. In the next section we will delineate our approach to the formulation of the stabilization energy.

\section{Form of the Stabilization energy}

In this section we will develop a quasi-optimal expression for the stabilization energy. For the stress analysis of linearly elastic anisotropic solids Krysl [4] proposed the constitutive matrix $\widehat{\mathbf{D}}$ that induces the stabilization strain energy $\widehat{\mathcal{U}}$ in the form

$$
\widehat{\mathbf{D}}=\phi \sum_{i=1}^{n_{m}} \psi_{i} K_{i} \boldsymbol{v}_{i} \boldsymbol{v}_{i}^{T}+\phi \sum_{j=n_{m}+1}^{6} K_{j} \boldsymbol{v}_{j} \boldsymbol{v}_{j}^{T}
$$


where $K_{i}$ and $\boldsymbol{v}_{i}$ are the eigenvalues and eigenvectors of the material stiffness matrix $\mathbf{D}$, and $0<\phi \leq 1$ and $0<\psi_{i} \leq 1$ are suitable scalar multipliers. The multipliers $\psi_{i}$ may be taken at a magnitude that scales the "stiff" moduli down to the magnitude of the "flexible" moduli. For instance, for an isotropic nearly incompressible material we take $n_{m}=1$ and $\psi_{1}=1-2 \nu$ as follows from the relationship between the Young's and bulk moduli. The multiplier $\phi$ was in Reference [4] chosen arbitrarily. Here we attempt to deduce how to rationally choose the stabilization amount in a quasi-optimal manner.

To develop our presentation, we shall consider application of the present element in the analysis of almost incompressible isotropic linearly elastic materials. (As we shall point out later, the assumption of isotropy is not required in general.) So we assume for the moment that the "real" material (as opposed to the "stabilization" material) properties are: $E$ of arbitrary positive value and $\nu \approx 1 / 2$. The moduli $K_{i}$ of the "real" material consist of one very large value (responsible for the volumetric locking), and five reasonably small moduli.

To design the "stabilization" material we could use formula (37), but here we propose to work with the elastic constants directly. Thus we introduce an isotropic stabilization material, which is defined by the Young's modulus and the Poisson ratio. The Young's modulus of the stabilization material can be taken as $\widehat{E}=E$, but the Poisson ratio must not be taken as $\widehat{\nu}=\nu$. Rather, we suggest taking

$$
\begin{aligned}
& \widehat{\nu}=\nu \quad \text { for } \nu \leq 0.3, \\
& \widehat{\nu}=(\nu+0.3) / 2 \quad \text { otherwise. }
\end{aligned}
$$

A few remarks:

1. Equation (38) clamps $\widehat{\nu}=\nu$ to a value less than or equal to 0.4 in order to avoid introducing volumetric locking to the stabilization material model.

2. This choice is equivalent to using $\psi_{1}=O(1-2 \nu)$ in (37).

3. The influence of this parameter on the convergence behavior is negligible as will be shown in the numerical examples below.

Now we shall consider the choice of the multiplier $\phi$. The main idea is that we could use this free parameter to improve the response of the hexahedral element in deformation modes that are different from uniform-strain deformations, i.e. in deformation modes that are only perceived by the full-quadrature stabilization. One such mode of deformation is pure bending.

Consider a rectangular brick under pure bending deformation in the plane $x-z$ as shown in Figure 1. The elastic energy can be estimated using the assumption that the cross-section does not change in shape or area as

$$
U=\frac{E I}{h_{x}} \alpha^{2} .
$$

Here the moment of inertia of the cross-section is

$$
I=\frac{h_{y} h_{z}^{3}}{12} .
$$



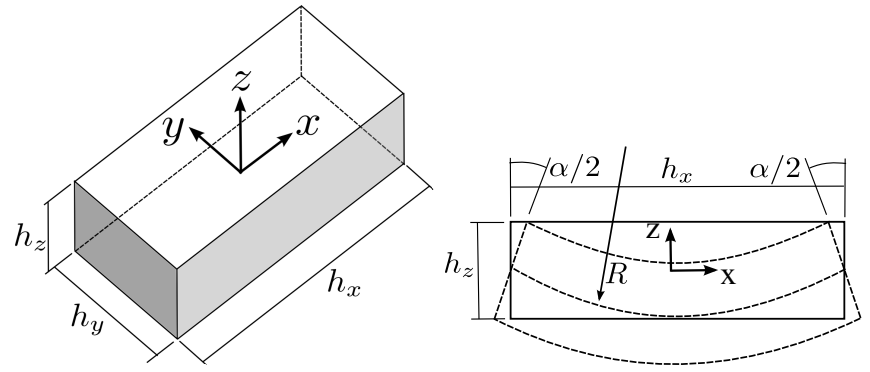

Figure 1: Sketch of a rectangular brick under pure planar bending

The hexahedron element subject to the same deformation pattern, that is the inclination of the two faces of the element that are orthogonal to the axis of the "beam" along $x$, stores the elastic energy

$$
U=\frac{E I}{h_{x}}\left[\frac{1+\Phi}{\Phi}\right] \alpha^{2},
$$

which includes both the energy of the stretches and the energy of the shear strains. Here we use the parameter well-known from the theory of shear-flexible beams

$$
\Phi=\frac{12 E I}{G A h_{x}^{2}}=2(1+\nu) \frac{h_{z}^{2}}{h_{x}^{2}} .
$$

In the limit of $h_{z} / h_{x} \rightarrow 0$, as the beam becomes more and more slender, the parameter $\Phi$ of Equation (42) will tend to zero, and hence the bending energy (41) will approach infinity and will be substantially in error. Clearly, we can match the bending energy of the hexahedron with the "exact" expression, if the (stabilization) material of the hexahedron is based on the reduced Young's modulus

$$
E^{*}=E \frac{\Phi}{1+\Phi}
$$

instead of the original $E$. In the context of the formulation (37) this is accomplished by taking the scaling parameter $\phi=\Phi /(1+\Phi)$ and therefore we suggest the stabilization material parameters be adopted as

$$
\widehat{E}=E \frac{\Phi}{1+\Phi}, \quad \begin{array}{ll}
\widehat{\nu}=\nu \quad \text { for } \nu \leq 0.3, \\
\widehat{\nu}=(\nu+0.3) / 2 \quad \text { otherwise. }
\end{array} .
$$

Evidently, the bending deformation could also be considered in the other two orthogonal planes, $x-y$ and $y-z$. Therefore, the estimate (42) may be generalized in a straightforward way

$$
\Phi=2(1+\nu) \frac{\min \left[h_{x}^{2}, h_{y}^{2}, h_{z}^{2}\right]}{\max \left[h_{x}^{2}, h_{y}^{2}, h_{z}^{2}\right]}
$$


to account for the need to "soften" the element for the most "flexible" plane of bending.

The final consideration before we look at numerical examples may be given to the general-shape hexahedra. In order to make the parameter as inexpensive to compute as possible, we may define local coordinate directions using the columns of the Jacobian matrix evaluated at the centroid of the element. (This may remind us of the equivalent parallelepiped of Kussner and Reddy [13].) Then, the lengths referenced in Equation (45) may be taken as the norms of the vectors which constitute the columns of the Jacobian matrix. The approximation is admittedly very crude (the local coordinate directions defined by the columns of the Jacobian matrix are not even orthogonal), but again, it is only to define the stabilization, does not affect convergence to the correct answer as the mesh is refined, and it is inexpensive to compute.

We also remark here that application of the presented stabilization approach to anisotropic materials will be taken up in Section 4.9 in the context of an example. Henceforth we can relinquish the assumption that the material of the element is isotropic.

Note that Equation (44) defines the stabilization material without any user input. All that is required are the properties of the actual material and measures of the geometry of the element (the lengths of the sides of the equivalent brick). We may note the similarity between the present treatment and that of Nadler and Rubin [14]: the Cosserat brick in their approach is stabilized by postulating 18 deformation modes and matching the stiffness of the element inhomogeneous deformations to heuristically determined optimal values, resulting in 18 stabilization elastic constants. In the present work, a single deformation mode is chosen, and consequently the formulation is much simpler.

The element formulation described herein will be labeled H8MSGSO (Hexahedral 8-node Mean-Strain Gauss-rule Stabilized with Optimized performance). In the next Section we will illustrate the remarkable performance of the present formulation on standard benchmarks.

\section{Numerical examples}

In the numerical examples in this section we compare the performance of the present formulation with the mean-strain elements implemented in the Abaqus finite element software. The element C3D8RH is the mean-strain element with default hourglass stabilization (i.e. the artificial stiffness stabilization) as proposed by Flanagan and Belytschko [5]. The element denoted C3D8RH(enh) in the present work is the mean-strain element with the enhanced (assumed strain) hourglass stabilization as proposed by Puso in [3]. The element C3D8I is the incompatible-mode hexahedron $[15,16,17,13]$. For simplicity we will refer to the comparison elements by the Abaqus codes (C3D $8 X)$. For some comparisons we will also draw on articles that describe other high-performance hexahedra and shell elements (citations are provided close to where they are needed).

A note on the solutions of the plate and shell problems: Point loads are certainly not admissible for elasticity problems, but they are commonly used 
and the displacements under point loads are often target quantities in plate and shell benchmarks. Hexahedral (and other continuum) elements are often used to produce solutions to plate and shell problems, and as common and appropriate for such structures, loads and supports are often applied at points or along edges. With the understanding that convergence cannot be achieved in the limit of infinite refinement in the vicinity of the point load, the hexahedral element results can be interpreted rationally, as many works cited in the present manuscript do.

\subsection{Clamped circular plate with concentrated force}

The clamped circular plate as studied by [18] is considered to begin with for three reasons. Firstly, as pointed out by Areias et al. it is a challenging problem because of the clamped condition along the approximated circular boundary. Secondly, we will demonstrate the dependence of the solution on the choice of the Poisson ratio of the stabilization material model. Thirdly, the concentrated force is a challenging loading for the stabilized hexahedron as it would tend to generate an hourglassing mode.

The plate was adopted in the configuration of [18], and it will suffice to say here that the radius to thickness ratio of 100 indicates that hexahedral elements applied to the solution of this problem might be expected to have some trouble converging quickly as the structure is quite thin.

The present element was used with 2, 3, 4, and 5 layers of elements per thickness. The enhanced-strain hexahedron HIS [18] was used with a single layer of elements, and the multiple-quadrature eight-node hexahedron HEXDS [19] was applied with two layers of elements per thickness. Figure 2 shows the convergence of the displacement of the point of application of the concentrated force to the reference analytical solution. The present element was used with three different values of the Poisson ratio $\widehat{\nu}$ used in the stabilization material model. There are actually three curves in the figure for these different Poisson ratios, but they are visually indistinguishable. This indicates that the choice of the parameter $\widehat{\nu}$ does not play a significant role in the behavior of the stabilized element. As similarly close results are obtained for the other examples presented below, we refrain from showing multiple curves for different choices of $\widehat{\nu}$ and only the value defined by equation (38) is used.

To address the other two points: Clearly the present element converges very well for this thin plate. It would be interesting to compare with the highperformance HIS element used with multiple layers through the thickness, but unfortunately that implementation is not available to us. Lastly, no hourglassing modes could be detected visually in the response of the plate when analyzed with the present formulation.

\subsection{Twisted beam}

The twisted clamped beam is a standard benchmark from the MacNealHarder set [20]. The coarsest mesh is $2 \times 1 \times 1$ (two elements lengthwise);

uniform refinement is used to produce finer meshes. The normalized deflection 


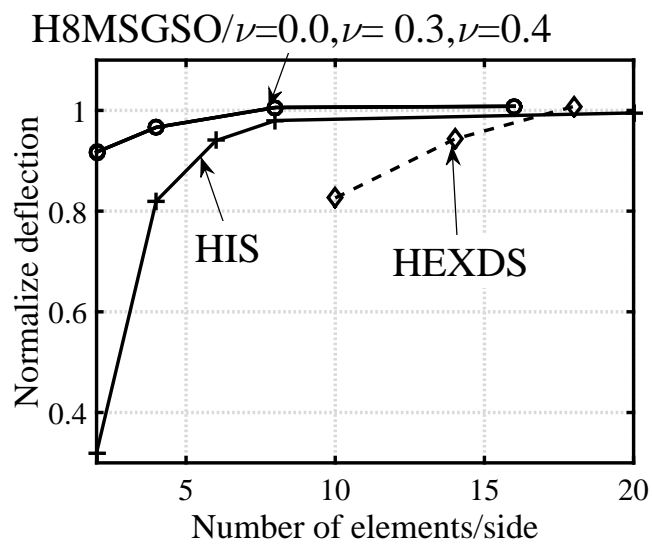

Figure 2: Clamped circular plate with a concentrated force at the center and radius to thickness ratio of 100. Normalized deflection. Key: HIS - enhanced-strain hexahedron [18], HEXDS - multiple-quadrature eight-node hexahedron [19], H8MSGSO - present hexahedron: results for three different values of the Poisson ratio $\hat{\nu}$ used in the stabilization material model.

data are shown in Figure 3 for both directions of applied load. The present formulation keeps up well with C3D8RH(enh) and with C3D8I; it does not cope well with the mesh consisting of a single element through the thickness, but its convergence is very satisfactory for two or more elements through the thickness. The enhanced-stabilization C3D8RH(enh) appears to be converging to the wrong answer.

\subsection{Simply-supported rhombic plate with $30^{\circ}$ angle, side-to-thickness ratio 1000}

The problem of the simply-supported rhombic plate is considered a challenging task for plate and shell elements due to the existence of moment singularities at the obtuse corners. The plate is relatively thin: side-length to thickness ratio of 1000. The thickness is uniformly divided into 1, 2 or 4 layers with the element edges parallel to the edges of the plate. The central deflection is computed and normalized by the series solution of Morley [21]

$$
w_{\text {center }}=0.408 \times 10^{-3} \frac{q L^{4}}{\frac{E t^{3}}{12\left(1-\nu^{2}\right)}} .
$$

where $q$ is the magnitude of the uniform load, $L$ is the length of the side of the plate, and $t$ is the thickness.

Figure 4 summarizes the results for the normalized deflection at the center of the plate. Figure 4(a) is for a single element through the thickness, and clearly all tested elements are substantially in error. Figure 4(b) is for two elements through the thickness. Both the present element and the enhancedstabilization hexahedron $\mathrm{C} 3 \mathrm{D} 8 \mathrm{RH}(\mathrm{enh})$ are much improved in accuracy, which is comparable for these two elements. Finally, in Figure 4(c) (four elements through the thickness) we compare the present element also with the assumedstress hybrid brick of Sze and Fan [22] and with the plate element MITC4 of 


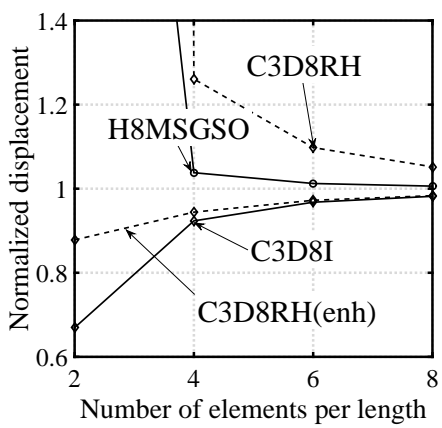

(a)

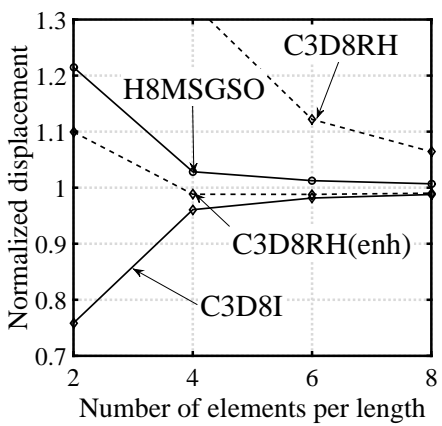

(b)

Figure 3: Twisted beam. Normalized deflection. (a) Loading in the direction of the $y$ axis. (b) Loading in the direction of the $z$ axis. Key: C3D8I - hexahedron with incompatible modes, $\mathrm{C} 3 \mathrm{D} 8 \mathrm{RH}$ - hybrid uniform-strain hexahedron with reduced integration and default hourglass stabilization, C3D8RH(enh) - hybrid uniform-strain hexahedron with reduced integration and enhanced hourglass stabilization, H8MSGSO - present element.

Bathe and Dvorkin [23]. While the assumed-stress brick is the best performer, the present element is not far behind, and both compare favorably with the specialized plate element. The error of the enhanced-stabilization C3D8RH(enh) is relatively small, but the element does not converge to the correct answer. The incompatible-mode brick C3D8I and the perturbation-stabilization hexahedron C3D8RH lock severely and are useless in this case.

\subsection{LE6 NAFEMS benchmark (stress in rhombic plate)}

This is a test recommended by the National Agency for Finite Element Methods and Standards (U.K.): Test LE6 from NAFEMS Publication TNSB, Rev. 3, "The Standard NAFEMS Benchmarks," October 1990. The support conditions and uniform distributed loading on the top surface of the plate is as for the rhombic plate discussed above. The edge length to thickness ratio is 100 in this case. The target quantity is the largest principal stress on the lower (traction-free) surface at the geometrical center of the plate. The target value is $0.802 \mathrm{MPa}$.

The plate was modeled with $4,8,16$, and 32 elements along the edge of the plate and a variable number of element layers through the plate thickness. Results for the present element are shown in Figure 5 for the elementwise values (as representative of the value of the stress within the layer of hexahedra adjacent to the free surface), and for the values of the principal stress extrapolated from the elementwise quantities to the free surface. Clearly the predictions of the extrapolated principal stress are fairly close to the reference quantity even for the coarsest mesh, with very slight dependence on the number of layers of elements through the thickness. 
(a)

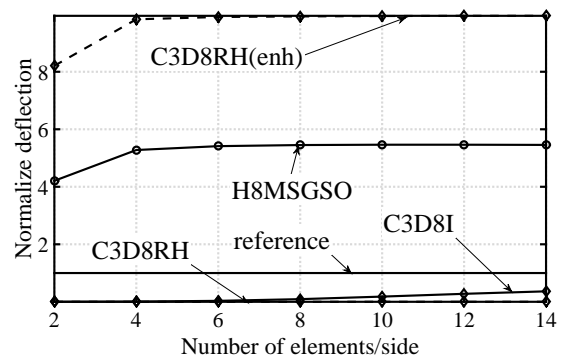

(b)

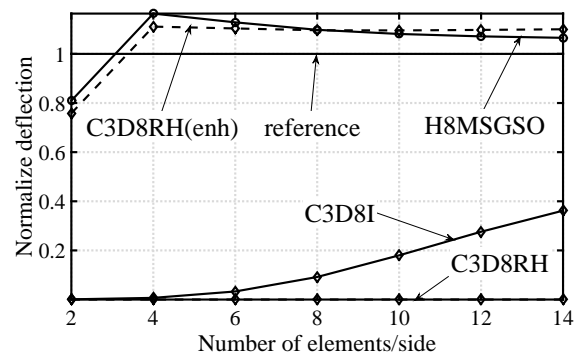

(c)

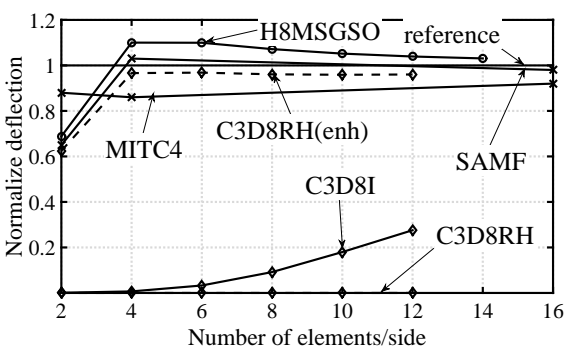

Figure 4: Normalized displacement at the center of a simply-supported rhombic plate with a $30^{\circ}$ angle. (a) Two layers of hexahedral elements through the thickness, (b) four layers of elements. Key: C3D8I - hexahedron with incompatible modes, C3D8RH - hybrid uniformstrain hexahedron with reduced integration and perturbation stabilization, C3D8RH(enh) hybrid uniform-strain hexahedron with reduced integration and enhanced stabilization, SAMF - assumed stress hybrid brick of Sze and Fan [22], MITC4 - plate element of the same name of Bathe and Dvorkin [23], H8MSGSO - present element. 


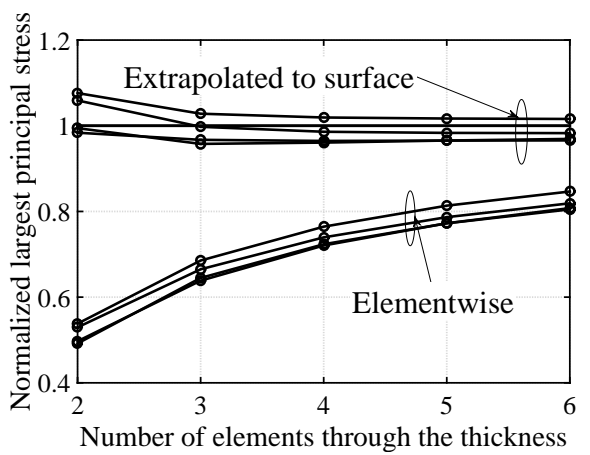

Figure 5: LE6 NAFEMS benchmark (stress in rhombic plate). Largest principal stress at the center of the bottom surface of the plate. Convergence of the element wise and extrapolated stress with increase of the number of layers of hexahedral elements through the thickness of the plate. Results for H8MSGSO - the present element - for the elementwise values (as representative of the value of the stress within the layer of hexahedra adjacent to the free surface), and for the values of the principal stress extrapolated to the free surface. Four curves are shown for $4,8,16$, and 32 elements along the edge of the plate.

\subsection{Hemispherical shell}

The full hemispherical shell with two pairs of pinching forces is a well-known benchmark for shell elements [20]. The mesh used in the present study is shown in Figure 6. The elements are rigorously tested in their ability to represent bending when distorted to a kite-like shape, especially in the coarse meshes. The meshes for the C3D8I and C3D8RH(enh) consist of a single element through the thickness. Increasing the number of elements through the thickness for these elements makes a negligible difference. On the other hand, the present element is used with one, two, and four elements through the thickness for the three results shown. (Use of multiple elements through the thickness is probably more representative of the actual use of the element, where stress resultants would also be of interest.) It is of interest to ascertain convergence of the present formulation that can be obtained with a fixed number of elements through the thickness.

In Figure 7 convergence was obtained for fixed number of layers through the thickness. The present element is actually stiffer with a single element through the thickness than when four elements through the thickness are used. The results happen to be most accurate for two layers for the thickness.

Figure 7 compares the present formulation with general-purpose hexahedra and also with specialized solid-shell elements. It is remarkable that the accuracy of the present element measures up well against the solid-shell elements [24, 25]. It would be interesting to find out how competitive the present hexahedron can be when accuracy versus cost are assessed, but that comparison was not possible in the present study.

The enhanced assumed strain (EAS) hexahedron of Caseiro et al. [26] is also quite accurate, but it is also much more expensive than the present for- 

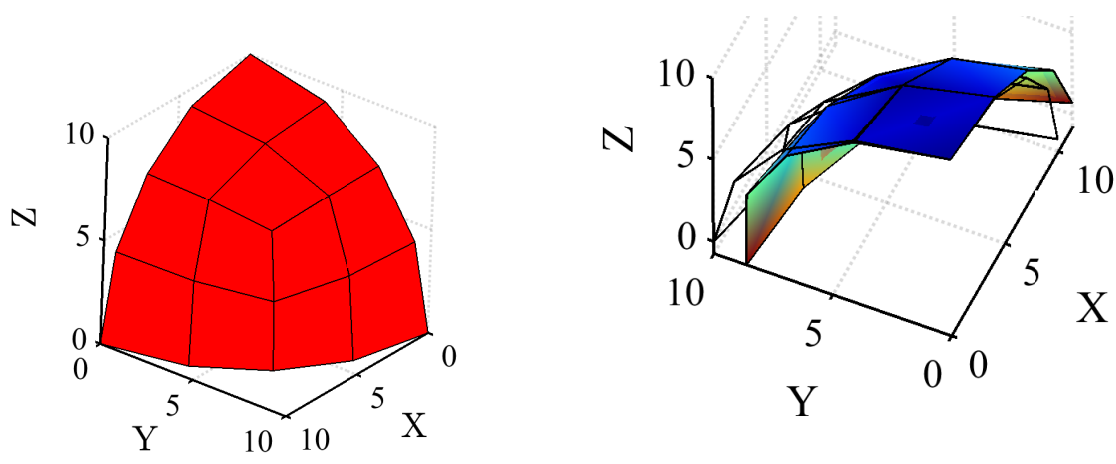

Figure 6: Pinched hemispherical shell. (a) The coarsest mesh used in the present study, with (b) the deflected shape (displacement magnified $20 \times$ ) shown.

mulation. The Abaqus hexahedra (incompatible-modes and enhanced assumed strain stabilization) are not particularly effective.

\subsection{Pinched cylinder}

This is another of the challenging benchmark problems from the MacNealHarder set [20]. The structure is a cylindrical shell closed off with rigid membranes. The shell is pinched with concentrated forces, and the output is the displacement underneath the force. Three symmetry planes are used to simplify the geometry (Figure 8(a)). Figure 8(b) shows the normalized displacement underneath the force. The perturbation-stabilized hexahedron C3D8RH locks, while the other comparison hexahedra perform similarly and converge well.

The present formulation is used with multiple layers of elements through the thickness. The initial mesh has just two elements through the thickness, and then uniform refinement is applied to produce subsequent meshes. The present H8MSGSO compares favorably with the high-performance element of Areias et al. [18] and the more reliable Abaqus hexahedra.

\subsection{Vibration modes and frequencies of squishy cube}

It is of considerable interest to develop the stabilization so that it is applicable in dynamic (vibration or wave propagation) problems as well as in statics. A guarantee that the formulation is stable in a static problem does not necessarily mean that the frequencies in dynamic problems are represented correctly.

Puso and Solberg [27] have discussed the ability of nodal integration to produce correct dynamic solutions using the example of a free-vibration spectrum of a unit cube of nearly incompressible isotropic material, $E=1, \nu=.499$, and $\rho=1$. The natural frequencies were presented in [27] for a mesh consisting of $16 \times 16 \times 16$ elements.

Here we use this example to illustrate two effects: (i) we show that the vibration spectrum is reproduced correctly (of course, only in this example and 


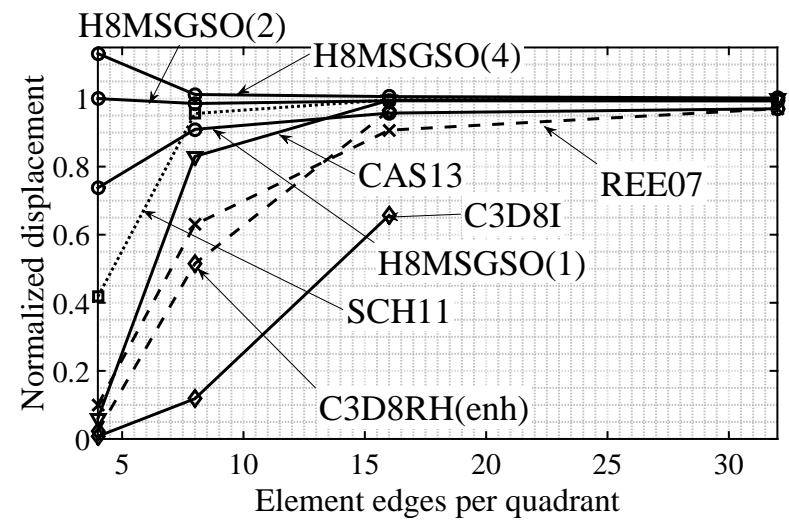

Figure 7: Hemispherical shell with pinching forces. Key: C3D8I - hexahedron with incompatible modes, C3D8RH - hybrid uniform-strain hexahedron with reduced integration and perturbation stabilization (locks severely, not shown), C3D8RH(enh) - hybrid uniform-strain hexahedron with reduced integration and enhanced stabilization, CAS13 - enhanced assumed strain hexahedron of Caseiro et al. [26], REE07 - reduced integration solid-shell element of Reese [24], SCH11 - reduced integration solid-shell element of Schwarze and Reese [25], $\operatorname{H8MSGSO}(n)$ - present hexahedron with $n$ layers of elements through the thickness.

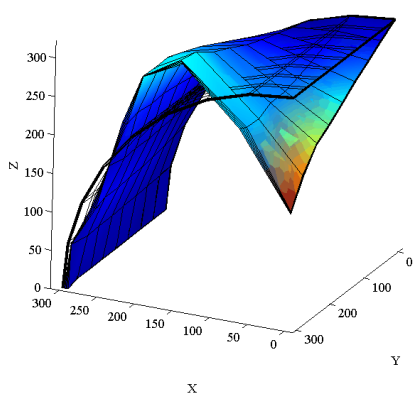

(a)

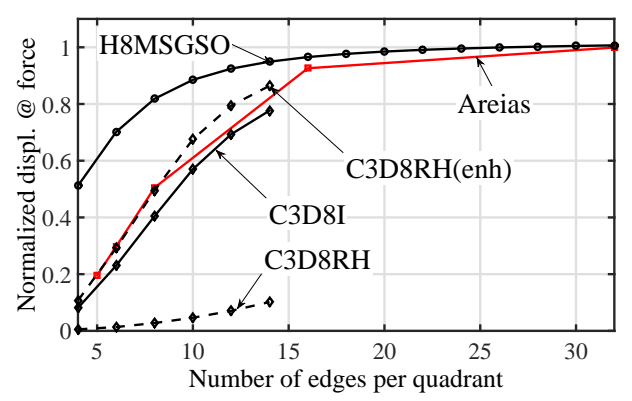

(b)

Figure 8: Pinched cylinder. Normalized deflection. (a) Mesh for eight element edges per quadrant. Deformed shape magnified $10^{7} \times$. (b) Normalized deflection under the pinching force. Key: C3D8I - hexahedron with incompatible modes, C3D8RH - hybrid uniform-strain hexahedron with reduced integration and default hourglass stabilization, C3D8RH(enh) - hybrid uniform-strain hexahedron with reduced integration and enhanced hourglass stabilization, Areias - enhanced-strain hexahedron of Areias et al. [18], H8MSGSO - present hexahedron. 


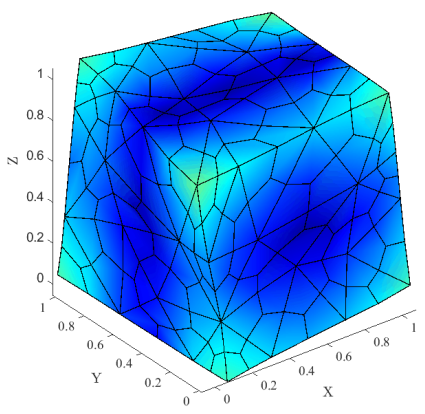

Figure 9: Vibration mode of squishy cube. Unstructured mesh for six element edges per side. The first non-rigid-body mode $(\approx 0.26[\mathrm{~Hz}])$.

for the 50 frequencies computed; we cannot say much more in general); (ii) we show the effect of element distortion.

First the natural frequencies are obtained with perfectly regular mesh. The same $16 \times 16 \times 16$ mesh as that used in [27] yielded the first six non-zero natural frequencies $0.2615,0.2615,0.3550,0.3550,0.3550,0.3606 \mathrm{~Hz}$ (for the lumped mass, obtained using the Hinton, Rock, Zienkiewicz algorithm [28]) and 0.2635, $0.2635,0.3590,0.3590,0.3590,0.3622 \mathrm{~Hz}$ (for the consistent mass). For comparison Puso and Solberg reported the first frequency as $0.262 \mathrm{~Hz}$ for the incompatible hexahedron (called QM6 in [27]), and $0.259 \mathrm{~Hz}$ for their stabilized nodal-integration tetrahedron.

The second mesh is constructed on purpose to be strongly suboptimal - the elements are highly distorted. The cube is first meshed with tetrahedra which are then converted to four hexahedra each. The mesh has (approximately) $6 \times$ $6 \times 6$ element edges per side. The mass matrix was adopted as lumped [28]. The first vibration mode (obtained with the present element) is shown in Figure 9.

The natural frequencies obtained with the present formulation and the C3D $8 X$ elements are compared with the reference values obtained with a structured $32 \times 32 \times 32$ mesh. Figure 10 shows (a) the first 44 nonzero frequencies, for the present element compared to the Abaqus hexahedra and the reference solution, and (b) the normalized error of the first 44 nonzero frequencies. It is evident that the stabilized hexahedra $\mathrm{C} 3 \mathrm{D} 8 \mathrm{RH}$ and $\mathrm{C} 3 \mathrm{D} 8 \mathrm{RH}(\mathrm{enh})$ are less accurate than the hexahedron with incompatible modes C3D8IH (hybrid version was required for the completion of the vibration study for this element due to the nearly incompressible response) and the present H8MSGSO hexahedron. Despite the very irregular shape of the elements, the present stabilization based on the norms of the columns of the Jacobian matrix at the centroid (the equivalent parallelepiped) seems to be working well.

\subsection{Rubber bushing}

This example demonstrates the quality of the computed stresses. A cylindrical rubber bushing of almost incompressible isotropic material, $E=3, \nu=$ 
(a)

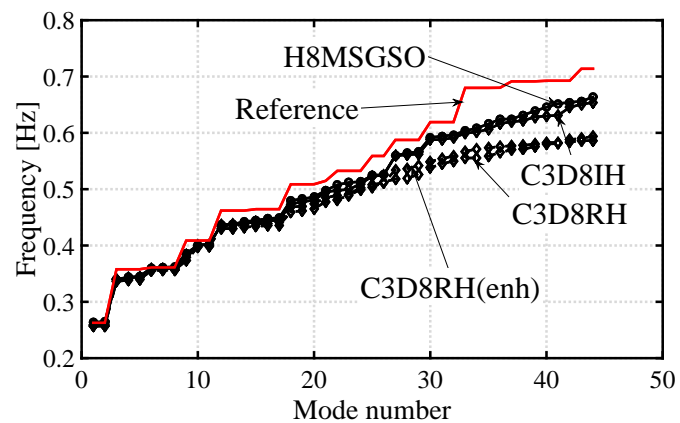

(b)

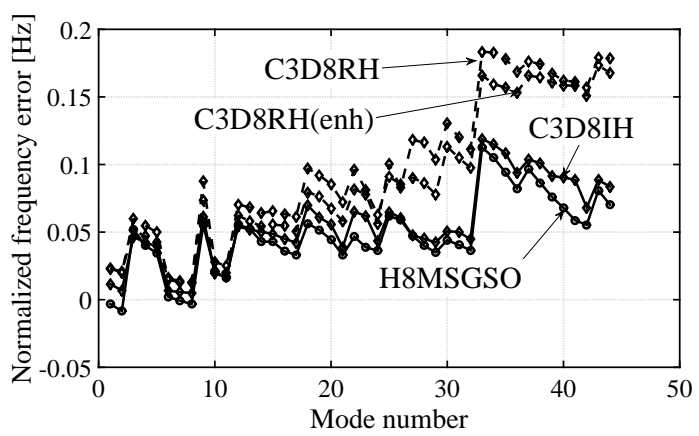

Figure 10: Vibration of squishy cube. (a) The first 44 nonzero frequencies. (b) Normalized error of the first 44 nonzero frequencies. Key: C3D8IH - hexahedron with incompatible modes, the hybrid (mixed) version, C3D8RH - hybrid uniform-strain hexahedron with reduced integration and default hourglass stabilization, C3D8RH(enh) - hybrid uniform-strain hexahedron with reduced integration and enhanced hourglass stabilization, H8MSGSO - present hexahedron. 


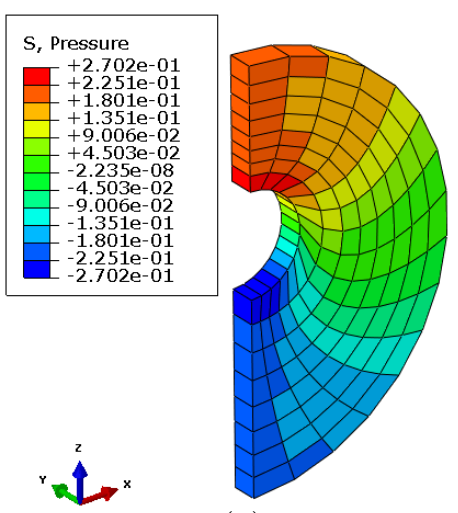

(a)

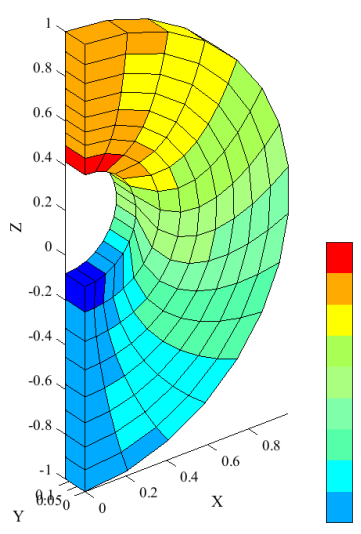

(b)

Figure 11: Rubber bushing. Regular mesh. Comparison of mechanical pressure distributions. (a) C3D8RH(enh) - hybrid uniform-strain hexahedron with reduced integration and enhanced hourglass stabilization, (b) H8MSGSO - present hexahedron. Displacements are magnified with a factor of 10 .

0.4999999, is loaded by prescribed translation of the interior cylindrical surface. The exterior radius is $R_{e}=1$, the interior radius is $R_{i}=0.25$, and the interior surface translates by $R_{i} / 15$ in the positive $z$ direction (that is upwards in Figures 11 and 12). The bushing is assumed to be under a plane strain condition, and therefore a slice of thickness $R_{i} / 2$ is modeled with zero through-the-thickness displacement applied on the annular sections. Symmetry boundary condition is also applied.

Figure 11 was generated with a regular mesh, and it shows the mechanical pressure color-coded on the deformed shape (magnified 10 times). The color maps are slightly different in the two software packages, but nevertheless the similar distribution of pressure for the C3D8RH(enh) (Abaqus hybrid uniformstrain hexahedron with reduced integration and enhanced hourglass stabilization) and for the present formulation can be appreciated. Note that both color scales are based on the same pressure range $-0.27 \leq p \leq 0.27$. Also of note is that the elementwise values of pressure are displayed (i.e. no nodal averaging was applied).

Next, an irregular mesh was generated of roughly the same number of elements by generating a tetrahedral mesh of a rectangular block, subdividing each tetrahedron into four hexahedra, and then mapping the rectangular block to the shape of the bushing. Consequently the hexahedra are very poorly shaped. Figure 12 again compares the mechanical pressure color-coded on the deformed shape, and rather similar distributions of pressure are obtained in this case as well as for the regular mesh. Finally a much finer mesh of the same unstructured type was generated and the results are shown in Figure 12(c): the well behaved nature of the pressure distribution can be appreciated. 


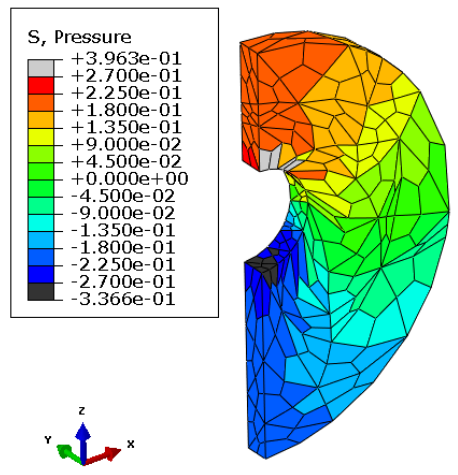

(a)

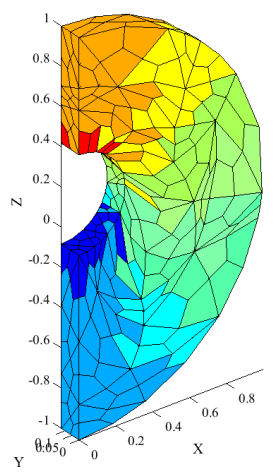

(b)

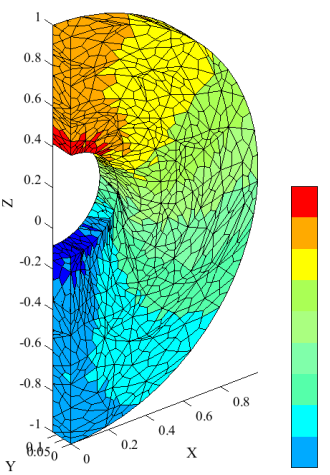

(c)

Figure 12: Rubber bushing. Irregular (unstructured) mesh. Comparison of mechanical pressure distributions. (a) C3D8RH(enh) - hybrid uniform-strain hexahedron with reduced integration and enhanced hourglass stabilization, (b) H8MSGSO - present hexahedron, (c) H8MSGSO - present hexahedron with much finer mesh. Displacements are magnified with a factor of 10 .

\subsection{Clamped beam with shear load, isotropic and orthotropic material}

We consider a cantilevered beam that is clamped at one end and loaded by a transverse shear force at the free end [29]. The $x$-axis is parallel to the beam's axis, the $z$-axis is vertically transverse. The dimensions are: width $W=1 \mathrm{~cm}$, length $L=9 \mathrm{~cm}$, and thickness $t=2 \mathrm{~cm}$. We consider both an isotropic material, and an anisotropic material with one of two different orientations of the local coordinate system. The anisotropic material is assumed to be a model suitable for a soft matrix reinforced with stiff uni-directional fibers. The elastic modulus along the stiff fiber is 100000 times higher than in the transverse directions, and the shear terms comparatively very small in magnitude. The anisotropic elastic properties are $E_{1}=100000 \mathrm{GPa}, E_{2}=E_{3}=1 \mathrm{GPa}, G_{12}=G_{13}=G_{23}=$ $0.2 \mathrm{GPa}$, and $\nu_{12}=\nu_{13}=\nu_{23}=0.25$. The fibers are oriented at an angle with respect to the beam's longitudinal axis as described by an orientation vector with components in the Cartesian coordinate system. The isotropic material is defined by $E=1 \mathrm{GPa}, \nu=0.25$.

The true maximum deflection of the beam in the direction of the load

$$
u_{z, \max }=\max _{\boldsymbol{x} \in \Omega}\left|\boldsymbol{u}_{z}(\boldsymbol{x})\right|
$$

was determined by a Richardson extrapolation from simulations with quadratic B-bar elements [29], and was used to normalize the displacement errors.

The mechanical response of the beam in terms of the estimated normalized true error of the maximum deflection is shown in Figure 14(a,b,c). The elements used to discretize the beam are elongated with an aspect ratio of 4.5 (Figure 13). This aspect ratio would significant the degrade the accuracy of linear elements that were without any enhancement of the bending stiffness. 
The behavior for isotropic compressible material is shown in Figure 14(a). As expected the Abaqus incompatible-mode C3D8I and the mean-strain elements C3D8RH perform rather well. The elements whose bending stiffness is not improved in any way, i.e. the Abaqus linear hybrid $\mathrm{C} 3 \mathrm{D} 8 \mathrm{H}$, the linear isoparametric element C3D8, and the B-bar hexahedron (H8-Bbar-ISO) [7] perform identically and poorly. The present formulation used with the quasioptimal value of the parameter $\phi$ is shown as H8MSGSO and it is clearly the most accurate element.

We shall now consider the stabilization of the hexahedron when the material is anisotropic. In this case, the material is orthotropic (more precisely, transversely isotropic). If that is the case, we see from the list of the input parameters that the $E_{1}$ modulus is very large compared to $E_{2}, E_{3}$. This introduces a constraint which may cause an element to lock, analogously to volumetric locking for isotropic materials [29]. As a simple variation on the ideas presented in Section 2, we could use an isotropic material to stabilize a hexahedron whose real material is anisotropic. Then the value of the Young's modulus to define the stabilization material could be adopted as

$$
\widehat{E}=\min \left[E_{1}, E_{2}, E_{3}\right] \frac{\Phi}{1+\Phi} .
$$

As before, the Poisson ratio of the stabilization material $\widehat{\nu}$ is determined from equation (44), where we take $\nu=0$ for any non-isotropic material. This quantity does not have a discernible effect on the accuracy or convergence behavior in this example either (similarly to the examples with isotropic materials). We wish to stress at this point again that the stabilization material is defined without any need for user intervention.

Now we look at the case of the orthotropic material with the fiber orientation $[\sqrt{2} / 2,-\sqrt{2} / 2,0]$ in Figure 14(b) and with the fiber orientation $[\sqrt{2} / 2,0,-\sqrt{2} / 2]$ in Figure 14(c). All input data are unchanged, including the mesh. The Abaqus incompatible-mode element C3D8I and one-point (reduced) integration hybrid elements C3D8RH are essentially as accurate as for the isotropic material. (The enhanced-stabilization C3D8RH(enh) is indistinguishable from C3D8I.) Importantly, with the stabilization parameters (48) the present formulation maintains its superior accuracy relative to the comparison elements.

\subsection{Plate with two orthogonal stiff reinforcing fibers}

Here we introduce a situation where the element response is constrained by stiff fibers. A similar example was studied by Krysl [4]. Rectangular thick plate of dimensions $W=0.1 \mathrm{~m}$ (width), $L=0.07 \mathrm{~m}$ (cantilevered length), and thickness $t=0.05 \mathrm{~m}$ is loaded by uniformly distributed shear traction of $1.0 \mathrm{kPa}$ at the free face parallel to the clamped face in the direction of the negative the $z$-axis - refer to Figure 15. The material of the plate is assumed to be a soft matrix reinforced with two orthogonal systems of stiff uni-directional fibers. Consequently, a homogenized orthotropic material model is assumed: The shear moduli and the Poisson ratios are taken as $G_{12}=G_{13}=G_{23}=0.2 \mathrm{GPa}$, 


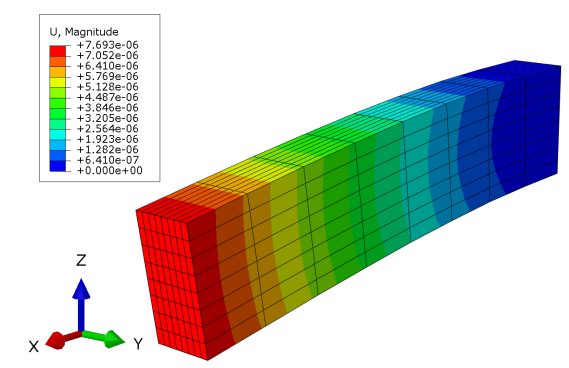

Figure 13: Clamped beam with shear load, isotropic and orthotropic material. Color-coded magnitude of displacement. Note the elongated elements with aspect ratio of 4.5 .

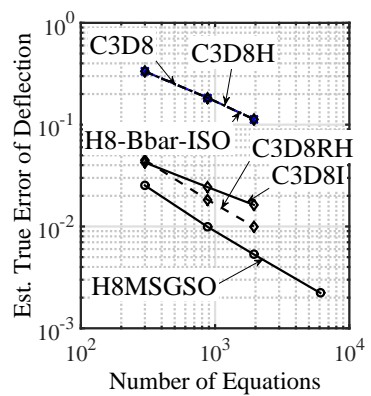

(a)

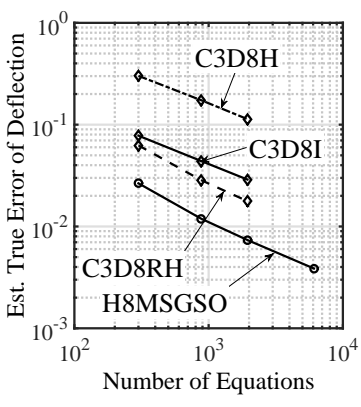

(b)

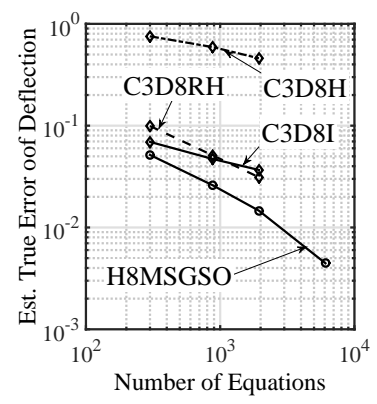

(c)

Figure 14: Displacement error of cantilever beam subject to shear load at the free end. The estimated true error of the maximum deflection: (a) Isotropic material; (b) Orthotropic material, stiff fiber aligned with $[\sqrt{2} / 2,-\sqrt{2} / 2,0]$; (c) Orthotropic material, stiff fiber aligned with $[\sqrt{2} / 2,0,-\sqrt{2} / 2]$. Key: C3D8H - linear hybrid hexahedron with uniform pressure, C3D8I - hexahedron with incompatible modes, C3D8RH - hybrid uniform-strain hexahedron with reduced integration, H8MSGSO - present hexahedron. 


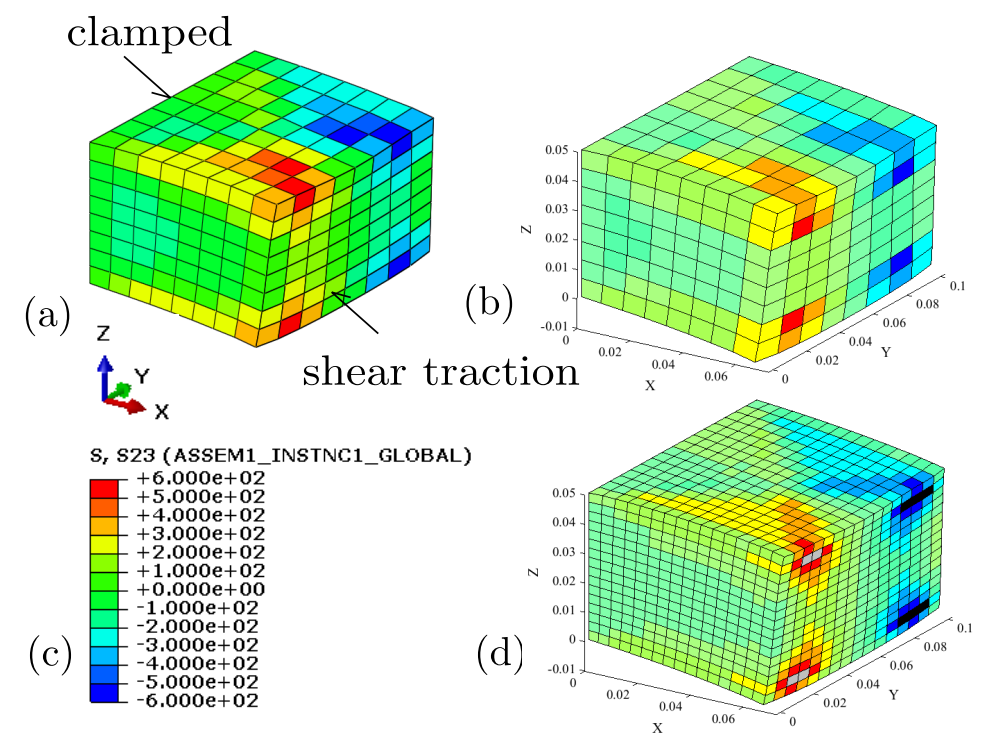

Figure 15: Plate with two orthogonal stiff reinforcing fibers. Elementwise stress $\sigma_{y z}$ in the global coordinate system. (a) C3D8RH element with the enhanced stabilization. (b) H8MSGSO element, same mesh as in (a). (c) Color bar common to all displayed results. (d) H8MSGSO element. Finer mesh.

and $\nu_{12}=\nu_{13}=\nu_{23}=0.25$ (we observe the definitions $\nu_{12} / E_{1}=\nu_{21} / E_{2}$ and $\left.\nu_{13} / E_{1}=\nu_{31} / E_{3}\right)$, the transverse Young's modulus $E_{3}=1 \mathrm{GPa}$, and the moduli along the fibers are $E_{1}=E_{2}=1000 \mathrm{GPa}$. The fiber systems are mutually orthogonal, located in the planes $z=$ const, and are oriented at $45^{\circ}$ with respect to the $x, y$ axes. The stabilization parameter has been again determined, without user intervention, from Equation (48).

Two systems of fibers are anchored in the clamped face. The constraints due to the restrained fibers make for an interesting distribution of stresses: observe the traction-loaded face where the fibers issuing from the clamped face are the focus of stress concentrations. As an example of the distribution of stresses in the global coordinate system we show the stress $\sigma_{y z}$ in Figure 15. Figure 15(d) shows the stress $\sigma_{y z}$ for refined mesh used with the present element. Figure 15(a) presents the stress $\sigma_{y z}$ for the enhanced-stabilization C3D8RH(enh) elements for a coarse mesh, while Figure 15(b) is for the present element and the same mesh. It is interesting to note that, compared with the fine-mesh solution, the distribution of stress on the coarse mesh is better captured with the present element than with the enhanced-stabilization $\mathrm{C} 3 \mathrm{D} 8 \mathrm{RH}(\mathrm{enh})$ element.

Figure 16 shows the convergence of the strain energy. The reference solution $1.214173 \times 10^{-6} \mathrm{~J}$ was calculated by Richardson extrapolation from results obtained with a 27 -node quadratic hexahedron instrumented with the generalized B-bar method (considering two constrained stiffness matrix modes) [29]. The elements C3D8RH(enh), and H8MSGSO converge similarly, but H8MSGSO has 


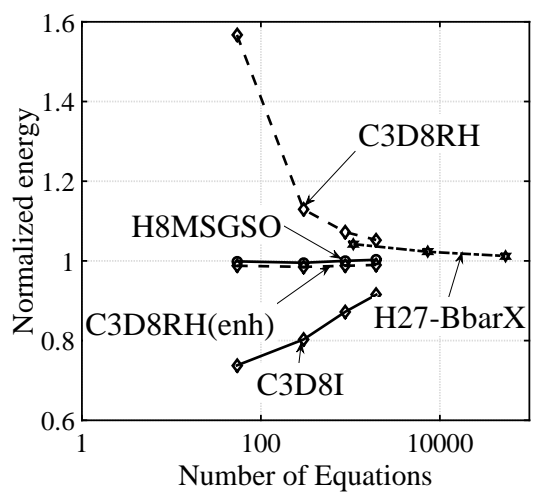

Figure 16: Plate with two orthogonal reinforcing fibers. Convergence of the normalized strain energy. Key: C3D8I - hexahedron with incompatible modes, C3D8RH - hybrid uniform-strain hexahedron with reduced integration and default hourglass stabilization, C3D8RH(enh) - hybrid uniform-strain hexahedron with reduced integration and enhanced hourglass stabilization, H8MSGSO - present mean-strain formulation, H27-BbarX - generalized B-bar formulation used with a 27-node hexahedron [29].

a slight edge. The default-stabilization C3D8RH and the incompatible-mode C3D8I exhibit much higher error.

\section{Conclusions}

A technique for stabilizing the mean-strain hexahedron was developed as a modification of the energy-sampling approach of Krysl [4]. The hourglassing modes of deformation are never explicitly separated from the rigid-body and mean-strain modes. Instead, stabilizing energy is postulated that is both added and subtracted from the strain energy of the element. The sampling of these two contributions is carried out by two different quadrature rules: the meanstrain quadrature is combined with the full Gauss quadrature rule. As a result, both consistency and full rank of the stiffness matrix (and hence convergence) are guaranteed.

For simplicity the developments are limited to linear elasticity, but with an arbitrarily anisotropic elasticity matrix. The stabilization energy is here assumed to be generated by a quadratic form whose coefficient matrix (i.e. the material constitutive matrix) is taken in the isotropic form and hence there are two input parameters to define. We develop an argument that avoids locking by fixing the value of the Poisson ratio away from $1 / 2$, and links the Young's modulus of the stabilization material to the input properties of the real material and (crucially) also to the geometry of the element. While the Poisson ratio can be chosen practically arbitrarily, the Young's modulus is matched to both the material and the geometry of the element. Utilizing the matching of the energy of the hexahedron in pure bending to a desirable value obtained from the analytical solution of pure bending, we come up with a reduced value of 
the Young's modulus that substantially increases the accuracy of the element in coarse-mesh bending applications. For orthotropic materials, a simple modification that considers the softest mode of deformation is found to work well.

As a result of these choices, the stiff part of the material constitutive matrix is only sampled by the mean-strain integration. In this way we are able to eliminate volumetric locking for isotropic materials as well as locking due to strongly anisotropic material properties, for instance for soft-matrix materials with unidirectional or bidirectional stiff fibers.

The accuracy and convergence characteristics of the present formulations compare favorably with the performance of uniform-strain elements as implemented in Abaqus. The present formulation also shines in comparison with the incompatible-mode hexahedron also available in Abaqus.

We further find that for thin structures the present element is quite accurate in comparison with specialized plate and shell elements. (A cost versus accuracy study was not performed, however.)

We do not mean to imply that the present element can be used for arbitrarily thin structures instead of plate or shell elements: rather, in the transition from fully solid sections to relatively thin plate- or shell-like sections the element can be robustly employed with aspect ratio (span to thickness) of 1000 or more. Hence, the present element can be an effective tool for relatively thin structures.

The element should not be employed with a single element through the thickness of the plate or shell. Rather, we wish to emphasize that in order to activate proper bending stiffness, at least two layers of elements are needed through the thickness of the structure. However, as described above, the element can be effectively employed with very large aspect ratios, which allows for the element to yield very good accuracy at relatively modest cost as meshing can be optimized to the shell-like geometry.

The original version of the present element [4] shared a sticky point with the perturbation (artificial stiffness) hourglassing stabilization [5]: there was an arbitrary parameter that needed to be set by the user. The present formulation removed such arbitrariness by linking the values of the stabilization parameters to the input parameters of the real material and to the shape of the element. Consequently there is no need for a user-specified parameter to stabilize the formulation. In addition to the good coarse-mesh accuracy, locking-free response, and robust performance for thin structures, this reinforces our confidence that the present element is suitable as a general purpose tool.

When constructing the element H8MSGSO, no assumptions are made as to the character of the "real" material constitutive matrix. Hence we believe that an arbitrarily nonlinear constitutive law can be accommodated. The stabilization parameters may need to be adjusted, for instance for softening or hardening materials. However, it is also possible that no such adjustment is necessary: further research is needed.

Since the stabilization strategy is based on energy, it is natural to pursue a line of thought that would extend the present technique to the fully nonlinear regime by defining an appropriate hyperelastic stabilization energy. Such an investigation was recently completed [30], with the result that the stabilization 
energy in the form of a neo-Hookean model works as well in the nonlinear regime of arbitrarily large strains as the linear isotropic elasticity for the present smallstrain applications. The total-Lagrangean approach to stabilization avoids the need to store stabilization forces, and problems with permanent deformation due to hourglassing hysteresis could be removed.

In conclusion, the increased coarse-mesh accuracy, resistance to the common kinds of locking for both massive and thin-walled structures, applicability in static and dynamic problems, and elimination of the need for additional user input to describe stabilization make the present mean-strain hexahedron with energy-sampling stabilization, H8MSGSO, an excellent general-purpose solid element.

\section{Acknowledgments}

Partial support from U.S. Navy CNO-N45, project management Frank Stone and Ernie Young, and continued support of Mike Weise (Office of Naval Research), is gratefully acknowledged.

[1] T. Belytschko, W. Liu, B. Moran, Nonlinear Finite Elements for Continua and Structures, Wiley: Chichester, West Sussex, 2000.

[2] W. J. T. Daniel, T. Belytschko, Suppression of spurious intermediate frequency modes in under-integrated elements by combined stiffness/viscous stabilization, International Journal for Numerical Methods in Engineering 64 (3) (2005) 335-353.

[3] M. A. Puso, A highly efficient enhanced assumed strain physically stabilized hexahedral element, International Journal for Numerical Methods in Engineering 49 (8) (2000) 1029-1064.

[4] P. Krysl, Mean-strain eight-node hexahedron with stabilization by energy sampling, International Journal for Numerical Methods in Engineering (2014) n/a-n/adoi:10.1002/nme.4721.

URL http://dx.doi.org/10.1002/nme.4721

[5] D. P. Flanagan, T. Belytschko, A uniform strain hexahedron and quadrilateral with orthogonal hourglass control, International Journal for Numerical Methods in Engineering 17 (5) (1981) 679-706.

[6] T. Belytschko, L. P. Bindeman, Assumed strain stabilization of the 8 node hexahedral element, Computer Methods in Applied Mechanics and Engineering 105 (2) (1993) 225-260.

[7] T. J. R. Hughes, Generalization of selective integration procedures to anisotropic and non-linear media, International Journal for Numerical Methods in Engineering 15 (9) (1980) 1413-1418. 
[8] S. Reese, On a physically stabilized one point finite element formulation for three-dimensional finite elasto-plasticity, Computer Methods in Applied Mechanics and Engineering 194 (45-47) (2005) 4685-4715.

[9] S. Reese, P. Wriggers, A stabilization technique to avoid hourglassing in finite elasticity, International Journal for Numerical Methods in Engineering 48 (1) (2000) 79-109.

[10] T. Belytschko, W. E. Bachrach, Efficient implementation of quadrilaterals with high coarse-mesh accuracy, Computer Methods in Applied Mechanics and Engineering 54 (3) (1986) 279-301.

[11] W. K. Liu, J. S. J. Ong, R. A. Uras, Finite-element stabilization matrices - a unification approach, Computer Methods in Applied Mechanics and Engineering 53 (1) (1985) 13-46.

[12] O. Zienkiewicz, R. Taylor, The Finite Element Method: The Basis, Oxford [etc.] : Butterworth Heinemann, 2000.

[13] M. Kussner, B. D. Reddy, The equivalent parallelogram and parallelepiped, and their application to stabilized finite elements in two and three dimensions, Computer Methods in Applied Mechanics and Engineering 190 (1517) (2001) 1967-1983. doi:10.1016/s0045-7825(00)00217-6.

[14] B. Nadler, M. B. Rubin, A new 3-D finite element for nonlinear elasticity using the theory of a Cosserat point, International Journal of Solids and Structures 40 (17) (2003) 4585-4614, times Cited: 41 Nadler, B Rubin, MB. doi:10.1016/s0020-7683(03)00210-5.

[15] J. C. Simo, M. S. Rifai, A class of mixed assumed strain methods and the method of incompatible modes, International Journal for Numerical Methods in Engineering 29 (8) (1990) 1595-1638.

[16] J. C. Simo, F. Armero, Geometrically nonlinear enhanced strain mixed methods and the method of incompatible modes, International Journal for Numerical Methods in Engineering 33 (7) (1992) 1413-1449. doi:10.1002/nme.1620330705.

[17] J. C. Simo, F. Armero, R. L. Taylor, Improved versions of assumed enhanced strain tri-linear elements for 3d-finite deformation problems, Computer Methods in Applied Mechanics and Engineering 110 (3-4) (1993) 359-386. doi:10.1016/0045-7825(93)90215-j.

[18] P. M. A. Areias, J. de Sa, C. A. C. Antonio, A. A. Fernandes, Analysis of 3d problems using a new enhanced strain hexahedral element, International Journal for Numerical Methods in Engineering 58 (11) (2003) 1637-1682. doi:10.1002/nme.835. 
[19] W. K. Liu, Y. Guo, S. Tang, T. Belytschko, A multiple-quadrature eightnode hexahedral finite element for large deformation elastoplastic analysis, Computer Methods in Applied Mechanics and Engineering 154 (1-2) (1998) 69-132, liu, WK Guo, Y Tang, S Belytschko, T.

[20] R. H. MacNeal, R. L. Harder, A proposed standard set of problems to test finite element accuracy, Finite Elements in Analysis and Design 1 (1985) 3 -20 .

[21] L. Morley, Skew plates and structures, Pergamon Press; [distributed in the Western Hemisphere by Macmillan, New York], 1963.

[22] K. Y. Sze, H. Fan, An economical assumed stress brick element and its implementation, Finite Elements in Analysis and Design 21 (3) (1996) 179 200. doi:10.1016/0168-874x(95)00038-u.

[23] K. J. Bathe, E. N. Dvorkin, A 4-node plate bending element based on Mindlin-Reissner plate-theory and a mixed interpolation, International Journal for Numerical Methods in Engineering 21 (2) (1985) 367-383. doi:10.1002/nme.1620210213.

[24] S. Reese, A large deformation solid-shell concept based on reduced integration with hourglass stabilization, International Journal for Numerical Methods in Engineering 69 (8) (2007) 1671-1716. doi:10.1002/nme.1827.

[25] M. Schwarze, S. Reese, A reduced integration solid-shell finite element based on the EAS and the ANS concept-geometrically linear problems, International Journal for Numerical Methods in Engineering 80 (10) (2009) 1322-1355. doi:10.1002/nme.2653.

[26] J. F. Caseiro, R. J. A. de Sousa, R. A. F. Valente, A systematic development of EAS three-dimensional finite elements for the alleviation of locking phenomena, Finite Elements in Analysis and Design 73 (2013) 30-41. doi:10.1016/j.finel.2013.05.006.

[27] M. A. Puso, J. Solberg, A stabilized nodally integrated tetrahedral, International Journal for Numerical Methods in Engineering 67 (6) (2006) $841-867$.

[28] E. Hinton, T. Rock, O. C. Zienkiewicz, A note on mass lumping and related processes in the finite element method, Earthquake Engineering \& Structural Dynamics 4 (3) (1976) 245-249. doi:10.1002/eqe.4290040305.

[29] S. P. Oberrecht, J. Novák, P. Krysl, B-bar FEMs for anisotropic elasticity, International Journal for Numerical Methods in Engineering 98 (2) (2014) 92-104. doi:10.1002/nme.4621.

[30] P. Krysl, Mean-strain eight-node hexahedron with optimized energy-sampling stabilization for large-strain deformation, International Journal for Numerical Methods in Engineering (2015) n/an/adoi:10.1002/nme.4907. 